incidence for the Irish population of transient pseudohypoaldosteronism was 1 per 13,200 live births per year for the study duration.

Conclusions Salt-wasting is a rare complication of UTI, especially if associated with underlying UTM. There is a similar annual incidence rate to the previously reported incidence of congenital adrenal hyperplasia in Ireland. Boys appear to be at particular risk. Prognosis is good if the condition is recognised and managed promptly.

\section{GP140 CYCLICAL CUSHING'S SYNDROME: A DIAGNOSTIC CHALLENGE}

'Deirdre O'Sullivan*, 'Rincy Koshy, Declan Cody 1,2. 'Our Lady's Children Hospital, Crumlin, Dublin, Ireland; ${ }^{2}$ University College Dublin, Dublin, Ireland

10.1136/archdischild-2019-epa.204

Aims Cyclic Cushing's syndrome is an uncommon disorder, defined by intermittent episodes of excess cortisol secretion. These episodes occur sporadically. The fluctuating clinical picture and conflicting biochemical findings make Cyclic Cushing's syndrome challenging to diagnosis. We report a case of Cyclic Cushing's syndrome is a 6 year old boy and discuss the challenges in diagnosis.

Methods A detailed chart review was performed. Data extracted from the medical records included presenting complaint, disease progression, laboratory results, imaging and clinical measurements.

Results At 4 years of age the patient presented with a two week history of rapid weight gain, increased appetite, lethargy, polydipsia and polyuria. The child has a background history of speech delay, obesity (weight $29 \mathrm{~kg},>99.6^{\text {th }}$ centile, BMI $23.3 \mathrm{~kg} / \mathrm{m}^{2}$ ), macrocephaly (OFC $56 \mathrm{~cm},>97^{\text {th }}$ centile), and facial freckling. During an in-patient stay, six hourly serum cortisol levels taken over a 48 hour period followed by a dexamethasone suppression test and a 24 hour urinary cortisol collection failed to support a diagnosis of Cushing's Syndrome. His significant facial freckling -with lip sparing and no mucosal involvement - prompted a Clinical Genetics referral. A diagnosis of Carney Complex (CNC) with a mutation in the PRKAR1A gene was made.

The patient continued to have episodes that would suggest episodic hyper-secretion of cortisol. Each episode lasted 3-4 weeks and then resolved. Parents reported 2 episodes in 2016, 1 episode in 2017 and 1 episode in 2018. In early 2018, the patient was admitted to hospital during an acute episode. A diagnosis of Cyclical Cushing's syndrome was confirmed by very elevated serum cortisol levels, elevated 24 hour urine free-cortisols, failure to suppress to dexamethasone and a very suppressed ACTH level during this admission. MRI and CT of abdomen however failed to reveal any adrenal lesions. Following discussions at multidisciplinary team meetings and with colleagues in adult Endocrinology, a decision was made to proceed with a bilateral adrenalectomy. The patient tolerated the procedure well. His adrenal histology was consistent with subtle changes suggestive of a mild Primary pigmented nodular adrenocortical disease (PPNAD) picture. Following adrenalectomy his symptoms have completely resolved but he will require lifelong Hydrocortisone and Fludrocortisone replacement.

Conclusion Our patient has a background history of CNC and PPNAD which has been linked in very occasional cases with Cyclical Cushing's syndrome. PPNAD is the most common endocrine manifestation of CNC. This case report highlights the difficulty in diagnosing Cyclic Cushing's syndrome.

\section{GP141 POSSIBLE AGGRAVATION OF DESMOID TUMOURS WITH PRIMARY OVARIAN FAILURE TREATMENT}

${ }^{1}$ Gillian O'Donnell ${ }^{*}$, 'Laura C McCarron, ${ }^{1,2}$ Ciara M McDonnell, ${ }^{1,3}$ Nuala P Murphy, ${ }^{1}$ Eirin Carolan. 'Department of Paediatric Endocrinology, Children's University Hospital, Temple St, Dublin, Ireland; ' ${ }^{2}$ iscipline of Paediatrics, University of Dublin, Trinity College, Dublin, Ireland; ${ }^{3}$ Discipline of Paediatrics, National University of Ireland, University College Dublin School of Medicine, Dublin, Ireland

\subsection{6/archdischild-2019-epa.205}

Introduction Chromosomal abnormalities are a common cause of primary ovarian failure in adolescents. Familial adenomatous polyposis (FAP) is an autosomal dominant predisposition to developing colonic polyposis arising from a germline mutation in the APC gene. Desmoid tumours (DT), otherwise known as 'deep fibromatosis', are locally invasive tumours that do not metastasize. DT develop in between 5-30\% of FAP carriers and are the second leading cause of death after colorectal carcinoma. Inductions of DT growth in both pregnancy and during oestrogen therapy have been reported. Selective oestrogen receptor modulators, including tamoxifen, are currently being utilised as a therapeutic agent for these tumours.

Case description A 14 year old girl was referred due to secondary amenorrhea and raised FSH levels. She entered puberty spontaneously, reached menarche at 13 years of age and had a regular 28 day menstrual cycle. She subsequently became amenorrheic 5 months later.

The index case was born at term by spontaneous uncomplicated vaginal delivery with a birth weight of $3.6 \mathrm{~kg}$. Subsequent failure to meet development milestones led to a hearing assessment at 9 months of age which diagnosed bilateral sensorineural hearing loss and bilateral cochlear implants were inserted. Genetics at that stage demonstrated a de novo chromosomal translocation involving the $\times$ chromosome and chromosome $15 \quad(46 \mathrm{X}$ translocation(X;15)(q13;q13).ishXq13 $($ Xist $\times 2)$ ).

Her father was subsequently found to be an FAP carrier and she is confirmed positive for this mutation. She has had annual surveillance colonoscopies; the most recent in June 2018 identified two adenomatous polyps. At 12 years of age she developed desmoid tumours, one located in the submandibular area and the other in the periumbilical area.

Investigations confirmed primary ovarian failure with undetectable oestradiol in the presence of elevated gonadotropins, normal androgens and low AMH levels. All other investigations were normal.

Discussion Oestrogen therapy is the mainstay of treatment in primary gonadal failure however, exogenous oestrogen is a risk factor in the exacerbation of desmoid tumour growth which could be life limiting. The family and their medical team need to strike a balance enabling optimisation of bone health without increased morbidity from tumour growth. Options were explored with the patient and her family with the ultimate decision to refrain from using exogenous sex hormone therapy with optimization of bone health. This is a complex case that poses therapeutic challenges in management and treatment goals of primary gonadal failure in the setting of desmoid tumours. 